\title{
Estudo prospectivo comparativo entre a endarterectomia e a angioplastia com stent e proteção cerebral no tratamento das lesões ateroscleróticas carotídeas: resultados em $\mathbf{3 0}$ dias
}

\author{
Prospective and comparative study between endarterectomy and stent angioplasty \\ with cerebral protection in carotid atherosclerotic lesions: 30-day results

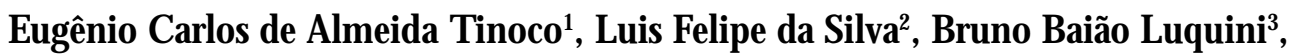 \\ Rafael Campanha', M arcelo N ascimento ${ }^{5}$, Luciana $\mathrm{H} \mathrm{orta}^{6}$
}

\section{Resumo}

O bjetivo: Analisar comparativamente os resultados, em 30 dias, entre a endarterectomia e a angioplastia com stent auto-expansível e filtro de proteção cerebral, avaliando a incidência de acidente vascular cerebral e óbito, bem como o tempo de permanência hospitalar no tratamento das lesões ateroscleróticas da bifurcação carotídea.

Método: Estudo prospectivo, em que foram tratados 80 pacientes, sintomáticos e assintomáticos, com lesões estenóticas maiores que 60 e $70 \%$, respectivamente, da bifurcação carotídea. 0 s pacientes foram divididos em dois grupos de 40 pacientes, que foram avaliados quanto a sexo, idade, comorbidades associadas e tabagismo.

Resultados: A taxa de acidente vascular cerebral e óbito foi de $5,0 \%$ em ambas as técnicas. 0 correu um caso $(2,5 \%)$ de ataque isquêmico transitório no grupo endovascular e nenhum na endarterectomia. No que se refere ao tempo de internação, o tratamento endovascular apresentou menor tempo em relação à endarterectomia, sendo estatisticamente significativo $(P<0,002)$.

C onclusões: N esta série apresentada, a incidência de acidente vascular cerebral e óbito em 30 dias foi de $5 \%$ nas duas técnicas utilizadas. Em relação ao tempo de internação, houve um menor tempo de permanência hospitalar a favor da técnica endovascular, com significância estatística $(P<0,002)$.

Palavras chaves: Carótida, endarterectomia, angioplastia.

1. Chefe, Serviço de Cirurgia Vascular e Endovascular, Hospital São José do Avaí (HSJA), Itaperuna, RJ.

2. Professor titular, Disciplina de Cirurgia Vascular, Universidade Federal do Rio de Janeiro (UFRJ), Rio de Janeiro, RJ.

3. Chefe de Clínica, Serviço de Cirurgia Vascular e Endovascular, HSJA, Itaperuna, RJ.

4. Residente (R2), Serviço de Cirurgia Vascular e Endovascular, HSJA, Itaperuna, RJ.

5. Residente (R1), Serviço de Cirurgia Vascular e Endovascular, HSJA, Itaperuna, RJ.

6. Residente (R2), Serviço de Cirurgia Geral, HSJA, Itaperuna, RJ.

Artigo submetido em 19.10.06, aceito em 30.12.06.

J Vasc Bras 2006;5(4):257-62.

Copyright $\odot 2006$ by Sociedade Brasileira de Angiologia e de Cirurgia Vascular.

\section{Abstract}

O bjective: To comparatively analyze the 30-day results between endarterectomy and angioplasty using self-expandable stent and filter protection in the treatment of carotid bifurcation atherosclerotic lesions. The primary endpoint was to analyze stroke and death rate, as well hospitalization time.

M ethods: Comparative and prospective study in 80 symptomatic and asymptomatic patients, with carotid bifurcation stenotic lesions greater than 60 and $70 \%$, respectively. The patients were divided into two groups of 40 and assessed according to gender, age, associated comorbid conditions and smoking.

Results: The stroke and death rate was $5.0 \%$ for both techniques. There was only one case of transient ischemic attack $(2.5 \%)$ in the endovascular group. Regarding hospital ization time, it was significantly lower in favor of the endovascular technique, with statistical significance $(P<0.002)$.

C onclusions: This study demonstrated a $5.0 \%$ incidence of stroke and death in 30 days considering both techniques. However, the endovascular group presented a statistically significant shorter hospitalization time than the endarterectomy group $(P<0.002)$.

Keywords: Carotid, endarterectomy, angioplasty.

\section{Introdução}

0 acidente vascular cerebral (AVC) isquêmico é a causa mais comum de déficit neurológico na população idosa, e a doença cerebral de origem extracraniana é responsável por aproximadamente $20-30 \%$ dos casos. A aterosclerose obliteranteéo principal fator etiológico dessas lesões.

A endarterectomia carotídea (EC), ao longo do tempo, apresenta-se como o principal método terapêutico no tratamento e prevenção desses acidentes encefá- 
licosisquêmicos. O sestudosN ASCET, ECST eACAS demonstraram a superioridade do tratamento cirúrgico sobre o clínico nas estenoses carotídeas. Baseado em dados desses estudos, o Ad H oc Committee of American $\mathrm{H}$ eart Association estabeleceu as indicações em que a endarterectomia deveria ser real izada ${ }^{1-4}$. Asprincipais indicações são pacientes sintomáticos com estenose $\geq 50 \%$ e em assintomáticos com estenose $\geq 60 \%$. Entretanto, a equipe cirúrgica deve ter morbimortalidade inferior a $6 \%$ nos pacientes sintomáticos, einferior a $3 \%$ nos assintomáticos.

0 desenvolvimento das técnicas endovasculares ea possibilidade de tratar as lesões carotídeas fizeram com que alguns autores propusessem a angioplastia neste território. 0 sresultadosiniciaisnão foram animadores, mas, com a introdução dos stents, houve uma melhora dos resultados, colocando a angioplastia com stent como uma real alternativa à endarterectomia.

\section{M etodologia estatística}

Para avaliar se houve diferença entre os grupos no que diz respeito a sexo, hipertensão, diabetes, doença coronariana, dislipidemias, tabagismo elado em quefoi aplicado o procedimento, realizou-se 0 teste do quiquadrado. N o caso em que ocorreram val oresesperados menores que 5, foi aplicado o teste exato de Fisher.

$\mathrm{N}$ a comparação entre as técnicas com relação à idadeedias deinternação, foi realizado o testedeM annW hitney, que tem como objetivo comparar amostras independentes em relação a uma medida de interesse. A lém disso, trata-sedeum testenão-paramétrico, isto é, não sebaseia namédiaedesvio padrão, esim nospostos/ posições (rank - posição do indivíduo na amostra) das medidas.

T odos os resultados foram considerados significativos para uma probabilidade de significância inferior a $5 \%(P<0,05)$, tendo, portanto, pelo menos $95 \%$ de confiança nas conclusões apresentadas.

\section{Pacientes e métodos}

No período de janeiro de 2004 a janeiro de 2006, foram tratados prospectivamente 80 pacientes no $\mathrm{H}$ ospital São José do Avaí (Itaperuna, RJ), divididos em dois grupos de 40 pacientes portadores de lesão aterosclerótica primária de carótidas.

Em ambos os grupos, os pacientes foram tratados pelo mesmo cirurgião.
A endarterectomia foi realizada sob anestesia locoregional, incisão longitudinal no pescoço efechamento primário da arteriotomia.

A angioplastia foi realizada através de acesso femoral, uso defiltro deproteção EPI/EZ (Boston Scientific) eW allstent (Boston Scientific).

O s critérios de inclusão foram: pacientes acima de 50 anos independente do sexo, lesões sintomáticas maioresque $60 \%$ eassintomáticas maiores que $70 \%$ ao D oppler colorido realizado pelo mesmo examinador.

O s critérios de exclusão foram: oclusão total da carótida interna; oclusão carotídea contralateral; história recente $(<1$ semana) de infarto agudo do miocárdio (IAM), ataqueisquêmico transitório (AIT) e/ou AVC; passado de qual quer intervenção no segmento carotídeo a ser tratado; lesão não aterosclerótica (radiação ou reestenose) e lesão no óstio da carótida comum ou lesão al ta da carótida interna que poupasse a bifurcação.

O s pacientes dos dois grupos tinham como exame pré-operatório o $\mathrm{D}$ oppler colorido de carótidas real izado pelo mesmo operador, bem como exames laboratoriais de rotina para esses pacientes, ou seja, hemograma, coagulograma, dosagem sérica de glicose, uréia, creatinina, sódio, potássio, colesterol etrigl icerídeos, além de eletrocardiograma (ECG) eecocardiograma para avaliação cardiológica. O s pacientes que porventura necessitavam de uma avaliação cardiológica mais rigorosa eram submetidos a teste ergométrico e, se necessário, coronariografia. As endarterectomias foram realizadas baseadas no D oppler colorido, ao passo que, no grupo do stent, realizava-se uma angiografia armada, que consistia na execução de um exame diagnóstico e terapêutico, caso se confirmasse a lesão.

D o ponto de vista farmacológico, os pacientes do grupo cirúrgico foram operados em uso deácido acetilsalićlico $100 \mathrm{~g} /$ dia e mantidos indefinidamente, ao passo que, no grupo da angioplastia, foi utilizado ácido acetilsalicílico $100 \mathrm{~g} /$ dia e ticlopidina $500 \mathrm{mg} / \mathrm{dia}$, iniciados 3 dias antes do procedimento, sendo ambos mantidos por 30 dias e posterior uso perene de ácido acetilsalicílico na dosagem de $100 \mathrm{mg} / \mathrm{dia}$.

N o grupo submetido a técnica endovascular, 45\% eram do sexo feminino e $55 \%$ do sexo masculino. N o grupo submetido a endarterectomia, 35\% eram do sexo feminino e $65 \%$ do sexo masculino. No entanto, não foram encontradas diferenças significativas entre as técnicas utilizadas no que diz respeito a sexo, com 
$P=0,361$. O bservamos que, naqueles submetidos a angioplastia, a idade média foi de 67,4 anos (52-83), enquanto nos submetidos a endarterectomia, foi de 69,5 anos (54-90), com $P=0,402$, sem diferenças significativas entre os dois grupos.

As duas técnicas estudadas apresentaram como principais comorbidades associadas a hipertensão arterial, diabetes melito, doença coronariana e dislipidemia. Entretanto, não houvediferençasignificativa entre as duas técnicas no que se refere a essas doenças, conforme descrito a seguir. A hipertensão arterial foi observada em $85 \%$ dos pacientes submetidos a angioplastia e em $80 \%$ dos submetidos a endarterectomia, com $P=0,556$. $Q$ uanto àincidência dediabetesmelito, foi detectada sua presença em $20 \%$ do grupo endovascular e em $30 \%$ nos submetidos a endarterectomia, sendo 0 valor de $P=0,302$. A incidência de doença coronariana foi de $25 \%$ no grupo endovascular versus $20 \%$ nos submetidos à endarterectomia, evidenciando 0 valor de $P=1,0$. A dislipidemia foi encontrada em $12,5 \%$ dospacientestratadoscom angioplastiaeem $5 \%$ dospacientessubmetidosa endarterectomia, com val or de $P=0,432$.

0 tabagismo estava presenteem $27,5 \%$ dos pacientes submetidos às duas formas de tratamento, sendo 0 valor de $P=1,0$.

$Q$ uanto ao lado em quefoi realizado o procedimento, o lado esquerdo foi acometido em $60 \%$ no grupo endovascular eem $42,5 \%$ naqueles tratados com endarterectomia com $P=0,117$.

Em relação à sintomatologia pré-operatória, também ocorreu um equilíbrio entreambasastécnicas. D os pacientessubmetidosatécnicaen dovascular, 70\% eram assintomáticos e 30\% apresentavam sintomas, sendo que destes, $41,7 \%$ apresentaram AIT e 58,3\% tiveram AVC. N o grupo da endarterectomia, $60 \%$ eram assintomáticos e $40 \%$ apresentavam sintomas; destes, 50\% haviam apresentado AIT e $50 \%$ tiveram AVC, com valor de $\mathrm{P}=0,587$.

A endarterectomia foi realizada através de incisão longitudinal na borda medial do esternocleidomastóideo. Imediatamente após a incisão, foi administrada heparina endovenosa na dose de $100 \mathrm{U} / \mathrm{kg}$. Atenção especial é dada na dissecção das estruturas, evitando a secção sem o seu reconhecimento. $N$ aslesões mais al tas, em que há necessidade de maior mobilização de segmento de carótida interna, real izamos a identificação e reparo da alça do nervo hipoglosso, evitando-se movimentos bruscos com o mesmo. 0 clampeamento foi realizado, iniciando sempre pelas carótidas interna, externa e comum. Foi realizada arteriotomia longitudinal, começando na carótida comum em direção à interna, não se estendendo além de $2 \mathrm{~cm}$, a não ser em casos necessários. U ma vez visualizada toda a placa, foi realizada endarterectomia convencional, ressecando as camadas íntima e média, deixando exclusivamente a adventícia. N os casos de diminuição do nível de consciência do paciente, utilizamos o shunt de Javid sob visualização direta dos lumens das carótidas interna e comum. Ao final da endarterectomia, procedemos à fixação distal da placa com fio de prolene 7-0, caso houvesse necessidade. 0 fechamento da arteriotomia foi feito da maneira tradicional, com prolene 6-0, iniciando sempre pela carótida interna e terminando 0 nó na carótida comum. Antes do final da sutura, foi realizada lavagem exaustiva da luz arterial com soro heparinizado, e subseqüentemente liberados os clamps arteriais na seguinte ordem: carótidas externa, comum e interna.

A angioplastia foi realizada em todos os casos através de anestesia local e punção de artéria femoral. A pós introdução debainha $5 \mathrm{~F}$, foram administrados $5.000 \mathrm{UI}$ de heparina pela bainha. A seguir, procedeu-se à realização da angiografia diagnóstica, que compreende 0 estudo das artérias carótidas e cerebrais. U ma vez realizado o diagnóstico da lesão carotídea e a intenção de tratá-la, procedemos à cateterização da carótida externa e à passagem de um fio guia 0,0035" extra-rígido, para trocarmos a bainha $5 \mathrm{~F}$ por uma bainha $6 \mathrm{~F}$ de $90 \mathrm{~cm}$ de comprimento ( $\mathrm{COOK}$, Bloomington, EUA), que foi introduzida até pouco antes da bifurcação carotídea. N este momento, foi administrada outra dose de heparina, mantendo-se, no total, a dosagem de $100 \mathrm{UI} / \mathrm{kg} .0$ próximo passo foi a liberação do filtro de proteção EPI/EZ (Boston Scientific, EU A), que deve ser sempre realizada em um segmento retilíneo da porção distal da carótida interna, para uma boa aposição do filtro na parede arterial, evitando assim a passagem de microêmbolos por fora do filtro. A seguir, procedeu-se à liberação do W allstent $(10 \times 24 \mathrm{~mm})$ (Boston Scientific, EU A). N ão realizamos pré-dilatação, reservando-a somente aos casos de lesão extremamente severa. $\mathrm{N}$ esses casos, utilizamos o balão de angioplastia coronária $4 \times 20 \mathrm{~mm}$. A pós a liberação do stent, 0 anestesiologista administrou atropina endovenosa na dose de $0,05 \mathrm{mg} / \mathrm{kg}$, independente da freqüência cardíaca do paciente. A pós-dilatação foi 
real izada com balão de angi oplastia que varia de 5,0$6,0 \times 20 \mathrm{~mm}$. Confirmado o resultado esperado da angioplastia, introduzimos a bainha para a captura do filtro de proteção, tomando-se o cuidado de não recolher completamente o filtro para o interior da bainha. Finalizamos o procedimento realizando uma angiografia carotídea e cerebral. U tilizamos rotineiramente o sulfato de protamina para reverter a metade da dose da heparina. A retirada da bainha é feita após a administração da protamina, sendo realizada compressão manual por 30 min no local da punção e curativo compressivo.

\section{Resultados}

0 uso do shunt foi necessário em 11 pacientes $(27,5 \%)$, sendo utilizado o shunt de J avid (T abela 1).

Tabela 1 - Freqüência do uso de shunt

\begin{tabular}{lcc}
\hline Shunt & $\mathbf{n}$ & $\%$ \\
\hline N ão & 29 & 72,5 \\
Sim & 11 & 27,5 \\
Total & 40 & 100,0 \\
\hline
\end{tabular}

0 tempo de clampeamento variou entre 20 e 45 min, com média de 27,1 e mediana de $27 \mathrm{~min}$.

0 filtro de proteção cerebral capturou debris em somente $11(27,5 \%)$ dos 40 pacientes submetidos a tratamento endovascular.

As complicações neurológicas (AVC) ocorreram em ambos os grupos, sendo dois pacientes $(5,0 \%)$ no grupo da en darterectomia eum $(2,5 \%)$ no grupo endovascular. 0 correu um óbito no grupo endovascular como conseqüência do IAM, e não houve óbito na cirurgia. H ouveum (2,5\%) caso de AIT no tratamento endovascular (T abelas 2 e 3 ).

Comparando-se as técnicas utilizadas em relação aos dias de internação, foi identificada uma significância estatística nos pacientes submetidos a endarterectomia, que permaneceram mais dias no hospital do que os pacientes submetidos a tratamento endovascular (T abela 4).
T abela 2 - Avaliação de complicações dos pacientes submetidos a angioplastia (acidente vascular cerebral eóbito)

\begin{tabular}{lcc}
\hline Variáveis & \multicolumn{2}{c}{ Freqüência } \\
\cline { 2 - 3 } & $\mathbf{n}$ & $\%$ \\
\hline Ausente & 38 & 95,0 \\
Presente & 2 & 5,0 \\
Total & 40 & 100,0 \\
\hline
\end{tabular}

T abela 3 - Avaliação de complicações relacionadas ao grupo de pacientes submetidos a endarterectomia (acidente vascular cerebral e óbito)

\begin{tabular}{lcc}
\hline Variáveis & \multicolumn{2}{c}{ Freqüência } \\
\cline { 2 - 3 } & $\mathbf{n}$ & $\%$ \\
\hline Ausente & 38 & 95,0 \\
Presente & 2 & 5,0 \\
Total & 40 & 100,0 \\
\hline
\end{tabular}

\section{D iscussão}

A realização de estudos randomizados e comparativos entre as duas técnicas foi necessária na busca da melhor técnica a ser empregada nos pacientes. 0 maior estudo publicado éo C AV AT AS (C arotid and Vertebral Artery Transuminal Angioplasty Study) ${ }^{5}$. Trata-se de um estudo multicêntrico randomizado que envolveu 560 pacientesem 24 centros. $D$ esses, 504 pacientescom estenose carotídea aptos a tratamento cirúrgico (253) ou endovascular (251) foram randomizados. 0 itenta e oito por cento no grupo endovascular e $91 \%$ no grupo cirúrgico apresentavam sintomas nos 6 meses queantecederam o estudo. N o grupo en dovascular, somente 55 (22\%) pacientes foram tratados com stent, enquanto 0 restante foi tratado somente com angioplastia. A incidência de AVC eóbito foi de $9,9 \%$ na endarterectomia e $10 \%$ no grupo endovascular, taxa excessivamentealta em relação aos resultados do N ASCET e ECST.

Subseqüentemente, alguns estudos comparativos foram realizados, porém 0 alto índice de complicação fez com que eles fossem terminados prematuramente. O s dois principais exemplos são: o estudo Leicester, no qual, após 17 pacientes envolvidos, cinco dos sete 
T abela 4 - M edidas descritivas e comparativas dos dias de internação hospitalar considerando a técnica utilizada

\begin{tabular}{lccccccc}
\hline & \multicolumn{8}{c}{ Medidas descritivas } \\
\cline { 2 - 8 } Variável & Téenica & $\mathbf{n}$ & M ínimo & M áximo & M édia & D P & P \\
\hline Dias & Stent & 40 & 1,0 & 8,0 & 2,0 & 1,4 & 0,002 \\
& Endart. & 40 & 1,0 & 9,0 & 2,8 & 1,9 & \\
\hline
\end{tabular}

DP = desvio padrão.

pacientes submetidos a angioplastia com stent sem proteção apresentaram déficit neurológico ${ }^{6}$; e o W allstent, estudo multicêntrico randomizado que foi terminado precocemente após o recrutamento de 219 pacientes, quando a incidência de AVC e óbito estava em $12,1 \%$ no grupo do stent sem proteção versus $4,5 \%$ no grupo da endarterectomia ${ }^{7}$. Em contraste a esses dois estudos, um estudo realizado em um único centro nos EU A randomizou 104 pacientescom estenosesintomática $>70 \%$, enão houve AV C tanto no grupo cirúrgico quanto no endovascular, ocorrendo um óbito na endarterectomia e nenhum no grupo do stent ${ }^{8}$. O SAPPH IRE (Stenting with Protection in Patientsat $\mathrm{H}$ igh Risk for Endarterectomy Study) é um estudo multicêntrico que comparou a eficácia da angioplastia com stent sob proteção cerebral em pacientes de al to risco cirúrgico à endarterectomia. Foram recrutados 334 pacientes, sendo 167 no grupo endovascular e 159 no grupo cirúrgico. Todos os pacientes eram sintomáticos com estenose $>50 \%$, ou assintomáticos com estenose $>80 \%$. Em relação à sintomatologia, 30 e $28 \%$ eram sintomáticos no grupo endovascular e cirúrgico, respectivamente. 0 objetivo primário do estudo era analisar a incidência cumulativa de eventos cardiovasculares maiores (AVC, IAM eóbito) em 30 diase 1 ano. A taxa de complicação em 30 diasfoi de 12,2 e20,1\% no grupo da angioplastia e cirurgia, respectivamente ( $P=0,004$ para não inferioridade e $P=0,053$ para superioridade $)^{9}$.

Em meta-análise realizada desses cinco estudos, de um total de 1.157 pacientes randomizados, não houve diferença significativa na taxa de AVC e óbito entre as duas técnicas, $8,6 \%$ no endovascular versus 7,1\% na cirurgia. D os três estudos que forneceram informação em relação à evolução do AVC, 6,0\% dos pacientes tratados com stent evoluíram para óbito ou apresentavam seqüelas severas versus $5 \%$ no grupo cirúrgico. Quando o IAM foi incluído, não houve diferença estatisticamentesignificativa entreosdoisgruposquando analisada a incidência de óbito, AVC eI AM , 9,0\% em ambos os grupos. N o que se refere à lesão nervosa, ocorreu em $7,2 \%$ na cirurgia e zero no grupo do stent $(P<0,00001)^{10}$.

Apesar dos resultados favoráveis ao tratamento endovascular demonstrado pelo SAPPH IRE, estudose registros com uso de stent e proteção cerebral apontam para um resultado com uma taxa de complicação relativamentealta. O ARCH eR (Acculink for Revasclarization in H igh Risk Patients) é uma série de três estudos multicêntricos, prospectivos, não-randomizados e seqüenciais, com fases distintas. A primeirafase envolveu 158 pacientes e não utilizou nenhum dispositivo de proteção cerebral. $\mathrm{Na}$ segunda fase, foi utilizado o protetor cerebral ACCUNET (Guidant Corporation, Santa C lara, Califórnia, EU A), que envolveu 278 pacientes. A terceira fase recrutou 145 pacientes e utilizou o mesmo protetor cerebral, porém com o sistema de troca rápida. Em todas as fases, foi utilizado o stent de nitinol ACCULINK (Guidant Corporation, Santa Clara, Califórnia, EU A). A taxa de óbito, AVC eIAM em 30 diasfoi de $8,3 \%$ (95\% deintervalo de confiança, $6,2-10,8 \%$ ), e de óbito e AVC foi de 6,9\% (95\% de intervalo de confiança, 5-9,3\%). A incidência em 30 dias de AVC maior ou fatal foi de $1,5 \%$. N ão houve AVC hemorrágico nesse grupo. A incidência entre 30 diase 1 ano de AVC ipsilateral foi de 1,3\%11.

D ois estudos publicados recentemente, o EVA$3 S^{12}$ e o SPACE ${ }^{13}$, não conseguiram demonstrar não inferioridade dos resultados do tratamento endovascular em relação à endarterectomia. São estudos randomizados prospectivos, que trataram pacientes sintomáticos com estenose $>70 \%$, de acordo com os critérios do ECST . Ambos foram suspensos, o primeiro devido ao alto índice de complicações no grupo da angioplastia $(9,6 \%)$ e o segundo, apesar do índice de complicações 
$(6,84$ versus $6,34 \%)$ semelhante, não foi suficientepara provar não inferioridadeestatística. Entretanto, asprincipais críticas aos estudos são que somente $27 \%$ dos pacientesutilizaram proteção cerebral no EVA-3S e, em relação à el egibilidade dos centros que participaram do estudo, questionou-se a curva de aprendizado dos realizadores do procedimento. Enquanto no EVA-3S era necessário o operador ter realizado 12 angioplastias com stent nas carótidas ou 35 angioplastias com stent no tronco supra-aórtico, sendo cinco no território carotídeo, no SPACE era necessário ter realizado, no mínimo, 25 angioplastias com ou sem stent nas carótidas com sucesso.

O CARESS (Carotid Revascularization using Endarterectomy or Stenting System) é um estudo clínico multicêntrico, prospectivo e não-randomizado, desenhado para determinar equivalência na taxa de AVC e óbito entrea angioplastia com stent e proteção cerebral e endarterectomia. 0 objetivo primário da fase I foi avaliar a incidência de AVC eóbito em 30 dias. Foram realizadas 254 endarterectomias e 143 angioplastias com stent, em pacientes sintomáticos (32\%) e assintomáticos (68\%). A taxadeAVC eóbito em 30 diasfoi de $3,6 \%$ no grupo cirúrgico e $2,1 \%$ no endovascular. Q uando foram analisadasa taxa de AVC, óbito eIAM , também não se observou diferença estatística entre as duas técnicas, 4,4\% na endarterectomia e 2,1\% na angioplastia ${ }^{14}$.

0 estudo mais aguardado é o CREST (Carotid Revascularization Endarterectomy versus Stent T rial), pois tem o suporte do $\mathrm{N}$ ational Institute of $\mathrm{N}$ eurological D isorders and Stroke e do $\mathrm{N}$ ational Institute of $\mathrm{H}$ ealth dos EU A. Esse estudo pretende comparar a eficácia da angioplastia com stent e proteção cerebral com a endarterectomia. É um estudo multicêntrico randomizado, em pacientes sintomáticos com lesões estenóticas $\geq 50 \%$. 0 s objetivos primários são avaliar a incidência deAVC, óbito el AM em 30 dias e AVC ipsilateral por um período de 4 anos de acompanhamento ${ }^{15}$.

\section{Conclusões}

$\mathrm{N}$ a série apresentada, a incidência de AVC e óbito em 30 dias foi de $5 \%$ em ambas as técnicas utilizadas neste estudo. Entretanto, no que se refere ao tempo de permanência hospitalar, houve uma redução estatisticamente significativa em favor da técnica endovascular.

\section{Referências}

1. Beneficial effect of carotid endarterectomy in symptomatic patients with high-grade carotid stenosis. N orth American Symptomatic Carotid Endarterectomy T rial Collaborators. N Engl J M ed. 1991;325:445-53.

2. MRC European Carotid Surgery Trial: interim results for symptomatic patients with severe (70-99\%) or with mild (0$29 \%$ ) carotid stenosis. European Carotid Surgery Trialists' Collaborative Group. Lancet. 1991;337:1235-43.

3. Endarterectomy for asymptomatic carotid artery stenosis. Executive Committee for the Asymptomatic Carotid Atherosclerosis Study. JAM A. 1995;273:1421-8.

4. M ooreW S, Barnett H J, BeebeH G, et al. G uidelines for carotid endarterectomy. A multidisciplinary consensusstatement from the $\mathrm{Ad} \mathrm{H}$ oc Committee, American Heart Association. Circulation. 1995;91:566-79.

5. Endovascular versus surgical treatment in patients with carotid stenosis in the Carotid and Vertebral Artery Transluminal Angioplasty Study (CAVATAS): a randomised trial. Lancet. 2001;357:1729-37.

6. N aylor AR, Bolia A, Abbott RJ, et al. Randomized study of carotid angioplasty and stenting versus carotid endarterectomy: a stopped trial. J V asc Surg. 1998;28:326-34.

7. Alberts MJ. Results of a multicenter prospective randomized trial of carotid artery stenting vs. carotid endarterectomy: a stopped trial. [abstract]. Stroke. 2001;32:325d.

8. BrooksWH, M CClure RR, J ones M R, Coleman TC, Breathitt L. Carotid angioplasty and stenting versus carotid endarterectomy: randomized trial in a community hospital. J Am Coll C ardiol. 2001;38:1589-95.

9. Yadav JS. C arotid stenting in high-risk patients: design and rationale of the SAPPH IRE trial. C leve Clin J M ed. 2004;71 Suppl 1:S45-6.

10. Coward LJ, Featherstone RL, Brown MM. Percutaneous transluminal angioplasty and stenting for carotid artery stenosis (C ochrane Review). Cochrane Libr. 2006:1.

11. Gray WA, H opkins LN, Yadav S, et al. Protected carotid stenting in high-surgical-risk patients: the ARCH eR results. J Vasc Surg. 2006;44:258-68.

12. M as JL, Chatellier $G$, Beyssen $B$, et al. Endarterectomy versus stenting in patients with symptomatic severecarotid stenosis. N Engl J M ed. 2006;355:1660-71.

13. SPACE CollaborativeG roup; Ringleb PA, AllenbergJ, et al. 30 day resultsfrom theSPACE trial of stent-protected angioplasty versus carotid endarterectomy in symptomatic patients: a randomised non-inferiority trial. Lancet. 2006;368:1239-47.

14. CARESS Steering Committee. Carotid revascularization using endarterectomy or stenting systems (CARESS): phase I clinical trial. J Endovasc Ther. 2003;10:1021-30.

15. Hobson RW 2nd. CREST (C arotid Revascularization Endarterectomy versus Stent T rial): background, design, and current status. Semin V asc Surg. 2000;13:139-43.

\section{Correspondência:}

Eugênio $C$ arlos de Almeida Tinoco

Rua Pastor Abelar Suzano de Siqueira, 305/603 -

Cidade N ova - CEP 28300-000 - Itaperuna, RJ

Tel./Fax: (22) 3824.3507

E-mail: ecatinoco@globo.com 